\title{
Uma prática de laboratório para equações diferenciais parciais: condução de calor em uma barra de cobre
}

\author{
A laboratory practice for partial differential equations: conducting \\ heat in a copper bar
}

Una práctica de laboratorio para ecuaciones diferenciales parciales: conducción de calor en una barra de cobre

Marcelo Lacortt ${ }^{1}$; Alexandre Pitol Boeira²; Anselmo Rafael Cukla ${ }^{3}$

\section{RESUMO}

Considerando que aulas práticas tornam o processo de ensino e aprendizagem mais agradáveis e significativos, assim como, buscando propor aos alunos um melhor entendimento do assunto equações diferenciais parciais, neste trabalho, foi realizado um experimento envolvendo ensaios de laboratório e o uso de equações diferenciais. A pesquisa em questão aborda um problema de condução de calor, o qual foi desenvolvido em dois momentos, o primeiro foi um ensaio em laboratório no qual foi realizado o aquecimento de uma barra de cobre. Já a segunda análise do problema foi abordada analiticamente, pela modelagem matemática da função condução de calor, a qual foi obtida pela equação diferencial parcial que descreve o fluxo de calor em uma barra. Dessa forma, o trabalho, em específico, consiste no estudo térmico realizado em uma barra de cobre cujas extremidades foram isoladas, e, durante o ensaio, a barra foi inicialmente aquecida e posteriormente resfriada até a temperatura ambiente, comparando assim os resultados obtidos via experimentação prática com os resultados obtidos pela solução analítica, através da equação condução de calor. Para coleta de dados no laboratório, foram utilizados sensores de temperatura e, para obtenção dos resultados pela equação condução de calor, foi utilizado o software Maple. De posse dos dados, foram feitas análises dos resultados e do comportamento, com o auxílio de tabelas e gráficos. Os resultados obtidos foram satisfatórios, com semelhanças significantes entre ambos os experimentos, implicando assim na viabilidade de aplicação da tarefa prática no desenvolvimento da disciplina.

Palavras-chave: Aulas práticas; Equações diferenciais parciais; Condução de calor.

\begin{abstract}
Considering that practical classes make the teaching and learning process more pleasant and meaningful, as well as, seeking to offer students a better understanding of the subject of partial differential equations, in this work an experiment was carried out involving laboratory tests and the use of differential equations. The research in question addresses a problem of heat conduction, which was developed in two stages, the first being a laboratory test where heating of a copper bar was performed. The second analysis of the problem was approached analytically, by the mathematical modeling of the heat conduction function, which was obtained by the partial differential equation that describes the heat flow in a bar. Thus, the work, in particular, consists of the thermal study carried out on a copper bar whose ends were insulated, and during the test the bar was initially heated and later cooled to room temperature, thus comparing the results obtained via practical

${ }^{1}$ Graduado em Matemática, Mestre em Engenharia e Professor do Instituto Federal Sul-rio-grandense (IFSul), Passo Fundo/RS - Brasil. E-mail: marcelolacortt@ifsul.edu.br

2 Doutor em Engenharia Mecânica e Professor Titular do Instituto Federal Sul-rio-grandense (IFSul), Passo Fundo/RS - Brasil. E-mail: alexandre.pitol@passofundo.ifsul.edu.br

3 Doutor em Engenharia Eletrotécnica e de Computadores e Professor Adjunto da Universidade Federal de Santa Maria (UFSM), Santa Maria/RS - Brasil. E-mail: cukla.anselmo@gmail.com
\end{abstract}


experimentation with the results obtained by the analytical solution, through the heat conduction equation. For data collection in the laboratory, temperature sensors were used, and to obtain the results through the heat conduction equation, the Maple software was used. With the results in hand, analyzes of the results and behavior were carried out, with the help of Tables and Graphs. The results obtained were satisfactory, with significant similarities between both experiments, thus implying the feasibility of applying the practical task in the development of the discipline.

Keywords: Practical classes; Partial differential equations; Conduction of heat.

\section{RESUMEN}

Considerando que las clases prácticas hacen más placentero y significativo el proceso de enseñanza y aprendizaje, así como, buscando ofrecer a los estudiantes una mejor comprensión del tema de ecuaciones diferenciales parciales, en este trabajo se realizó un experimento que involucró pruebas de laboratorio y el uso de ecuaciones. La investigación en cuestión aborda un problema de conducción de calor, que se desarrolló en dos etapas, siendo la primera una prueba de laboratorio donde se realizó el calentamiento de una barra de cobre. El segundo análisis del problema se abordó analíticamente, mediante la modelización matemática de la función de conducción de calor, que se obtuvo mediante la ecuación diferencial parcial que describe el flujo de calor en una barra. Así, el trabajo, en particular, consiste en el estudio térmico realizado sobre una barra de cobre cuyos extremos fueron aislados, y durante la prueba la barra fue inicialmente calentada y luego enfriada a temperatura ambiente, comparando así los resultados obtenidos mediante la experimentación práctica con los resultados obtenidos por la solución analítica, a través de la ecuación de conducción de calor. Para la recolección de datos en el laboratorio se utilizaron sensores de temperatura y para obtener los resultados a través de la ecuación de conducción de calor se utilizó el software Maple. Con los resultados en la mano, se realizaron análisis de los resultados y comportamiento, con la ayuda de Tablas y Gráficos. Los resultados obtenidos fueron satisfactorios, con importantes similitudes entre ambos experimentos, lo que implica la viabilidad de aplicar la tarea práctica en el desarrollo de la disciplina.

Palabras clave: Clases prácticas; Ecuaciones diferenciales parciales; Conducción de calor.

\section{INTRODUÇÃO}

Com o objetivo de tornar as aulas de equações diferenciais parciais mais interessantes, assim como tornar a aprendizagem de tal assunto mais significante, esta pesquisa consiste em uma prática laboratorial, cujos resultados obtidos são comparados com os resultados obtidos pela equação condução de calor.

A motivação pela pesquisa se deu ao ministrar a disciplina de equações diferenciais parciais, na qual os alunos sugeriram uma aplicação de tal conteúdo em uma situação verídica, comparando assim os resultados práticos com os estudados em sala de aula. Para isso, inicialmente foi realizado um estudo piloto, com o objetivo principal de verificar a viabilidade ou não da realização da tarefa.

Levando em conta a importância da disciplina de equações diferenciais parciais, pois há emprego em diversas áreas, uma aplicação prática, envolvendo laboratórios e uso de tecnologias, pode ampliar a visão da utilização de tais conceitos.

Segundo Diacu (2004), a teoria das equações diferenciais começou a ser utilizada nas ciências físicas, expandindo-se, ao longo do tempo, para as demais áreas, desde engenharia, biologia, medicina, negócios, até as artes.

A pesquisa foi realizada em um laboratório de física, entre um grupo de professores do Instituto Federal Sul-rio-grandense, utilizando e operando com os materiais de maneira criativa e improvisada. 
Com a cooperação e o envolvimento dos participantes no experimento, tivemos resultados interessantes e que certamente são viáveis no desenvolvimento da disciplina, assim como contribuirão positivamente para o entendimento do estudo das equações diferenciais parciais.

\section{FUNDAMENTAÇÃO TEÓRICA}

Para desenvolver a pesquisa, foi necessário um resgate teórico em assuntos que nortearam o referido estudo, baseado em autores que tratam sobre conceitos de aulas práticas, modelagem matemática, equações diferenciais parciais de segunda ordem e softwares Maple e Excel.

\subsection{Aulas práticas}

Aulas tradicionais, com metodologias convencionais, em que o professor cumpre o conteúdo proposto pelos ementários, sem se preocupar com a absorção de conhecimentos do aluno, somente com o comprimento dos conteúdos, estão se tornando algo ultrapassado no ensino, resultando em alunos desmotivados, desatentos e com baixo rendimento escolar. Sendo assim, aulas que envolvam práticas podem propor ao aluno um novo método de obtenção de conhecimento.

No ensino tradicional, o objetivo de estudo se apresenta quase sempre bem delineado, obedecendo a uma sequência predeterminada, com um objetivo final muito claro que, muitas vezes, nada mais é que "cumprir o programa da disciplina"! Ora, ensinar a pensar matematicamente é muito mais que isso. Portanto, é imprescindível mudar métodos e buscar processos alternativos para transmissão e aquisição de conhecimentos. (BASSANEZI, 2015, p. 11).

Com o objetivo de tornar as aulas de cálculo mais interessantes, procurando, em específico, motivar os alunos ao estudo de equações diferenciais, a pesquisa se baseia em uma proposta de emprego de aulas práticas. Para tal, é realizada uma fundamentação teórica em autores que apoiam esse método de ensino.

Para Lunetta (1992), as aulas práticas desempenham um papel importante de interação no desenvolvimento de conceitos científicos, além de permitirem que os estudantes aprendam como abordar objetivamente o seu mundo e como desenvolver soluções para problemas complexos. Já Hodson (1994) relata que atividades em que os alunos estejam ativos e não passivos são meios eficientes para melhorar o aprendizado, fortalecer explicações teóricas, reforçar informações de textos didáticos, além de levarem à construção de aprendizagens mais significativas.

Nesse sentido, aulas práticas podem criar possibilidades para que o aluno se torne mais produtivo e ativo, além disso, justificam o estudo teórico da sala de aula.

De acordo com Freire (1996), saber que ensinar não é transferir conhecimento, mas criar as possibilidades para a sua própria produção ou construção. Ou seja, ao criarmos novas possibilidades de aprendizagem, estamos ofertando novos caminhos para que o aluno seja autônomo na produção de conhecimento.

\subsection{Modelagem Matemática}

Para a realização de atividades práticas, que envolvam problemas matemáticos, um assunto indispensável a ser tratado é a modelagem matemática, pois esta descreve o processo como um todo, que vai desde a definição do problema, até a resolução e a validação dos resultados. 
Segundo Bassanezi (2004), a modelagem matemática é um "método", com etapas previamente definidas. Para o autor, a modelagem de uma situação problema ou de um problema real deve seguir uma sequência de etapas. Etapas estas que são: a experimentação, parte em que se processa a obtenção dos dados; a abstração, procedimento que deve levar à formulação dos modelos matemáticos; a resolução, substituição da linguagem natural pela linguagem matemática; e a validação, que é o processo de aceitação ou não do modelo proposto.

Para a realização de uma modelagem matemática, é necessário um modelo matemático. Segundo Lacortt,

[...] um modelo matemático é uma representação abstrata, ou uma aproximação, de um problema real. Tal representação pode ser expressa em termos de expressões matemáticas (equações), por meio de uma série de células inter-relacionadas em uma planilha de cálculo, entre outras. Em qualquer que seja o caso, o propósito de um modelo matemático é representar a essência de um problema de forma concisa. Isso traz uma série de vantagens, como permitir ao analista uma melhor compreensão do problema em estudo. (LACORTT, 2011, p. 23).

Sendo assim, a modelagem matemática consiste no processo matemático elaborado a partir do estudo de um problema real, o qual tem como finalidade descrever a realidade através de um modelo matemático abstrato e, assim, obter suporte para sua representação simplificada, solução e análise (GOLDBARG; LUNA, 2005).

\subsection{Equações diferenciais parciais de segunda ordem}

Considerando que esta pesquisa foi baseada na análise de condução de calor em uma barra de cobre, por dados resultantes de um estudo prático e comparado por resultados adquiridos pela equação condução de calor, obtida por uma equação diferencial parcial de segunda ordem, faz-se necessário um breve resgate das definições das equações diferenciais parciais.

As equações diferenciais parciais (EDP) são equações envolvendo duas ou mais variáveis, $x, y, z, t, \ldots$ , e derivadas parciais de uma função (variável independente), $u=u(x, y, z, t, \ldots)$ (IÓRIO, 2016).

Uma EDP com $n$ variáveis independentes $x_{1}, x_{2}, \ldots, x_{n}$ pode ser denotada de maneira genérica como:

$$
F\left(x_{1}, x_{2}, \ldots, x_{n}, u, \frac{\partial u}{\partial x_{1}}, \ldots, \frac{\partial u}{\partial x_{n}}, \frac{\partial^{2} u}{\partial x^{2}{ }_{1}}, \ldots, \frac{\partial^{2} u}{\partial x_{1} \partial x_{n}}, \ldots, \frac{\partial^{k} u}{\partial^{k} x_{n}}\right)=g\left(x_{1}, x_{2}, \ldots, x_{n}\right)
$$

Em que $k$ denota a ordem da derivada mais alta presente na equação e $g\left(x_{1}, x_{2}, \ldots, x_{n}\right)$ é o termo da equação que só depende das variáveis independentes.

Já as EDPs de segunda ordem são equações compostas por variáveis descritas por derivadas parciais de funções de duas variáveis independentes, podendo ser definida de forma genérica:

$$
F\left(x_{1}, x_{2}, u, \frac{\partial u}{\partial x_{1}}, \frac{\partial u}{\partial x_{2}}, \frac{\partial^{2} u}{\partial x_{1}{ }_{1}}, \frac{\partial^{2} u}{\partial x_{1} \partial x_{2}}, \frac{\partial^{2} u}{\partial^{2} x_{2}}\right)=g\left(x_{1}, x_{2}\right)
$$

Para resolver uma EDP de segunda ordem, o método mais utilizado é a separação de variáveis (NAGLE; SAFF; SNIDER, 2012). Essa técnica resulta em uma função $u\left(x_{1}, x_{2}\right)$, a qual pode ser escrita 
como o produto das funções $X_{1}\left(x_{1}\right)$ e $X_{2}\left(x_{2}\right)$, sendo que cada uma depende de uma variável independente, isto é, a solução será:

$$
u\left(x_{1}, x_{2}\right)=X_{1}\left(x_{1}\right) \cdot X_{2}\left(x_{2}\right)
$$

A partir de uma EDP de segunda ordem, obtém-se a equação do calor, a qual determina o campo de temperatura, ou seja, representa como a temperatura varia com a posição no meio (INCROPERA et al., 2008, p. 44).

A conceitualização da equação do calor, para Melo (2011), expressa um equilíbrio físico fundamental, no qual a taxa de calor que entra em qualquer parte da barra é igual à taxa de absorção de calor naquela parte da barra.

Para estudarmos o fenômeno da transmissão do calor e a equação diferencial parcial que modela o problema, convencionalmente, considera-se uma barra uniforme, de comprimento $L$, isolada termicamente nas superfícies laterais e com seção transversal pequena. Exemplo de uma barra de comprimento L está descrita na Figura 1.

Figura 1: Exemplo geométrico de uma barra para estudo de condição de calor

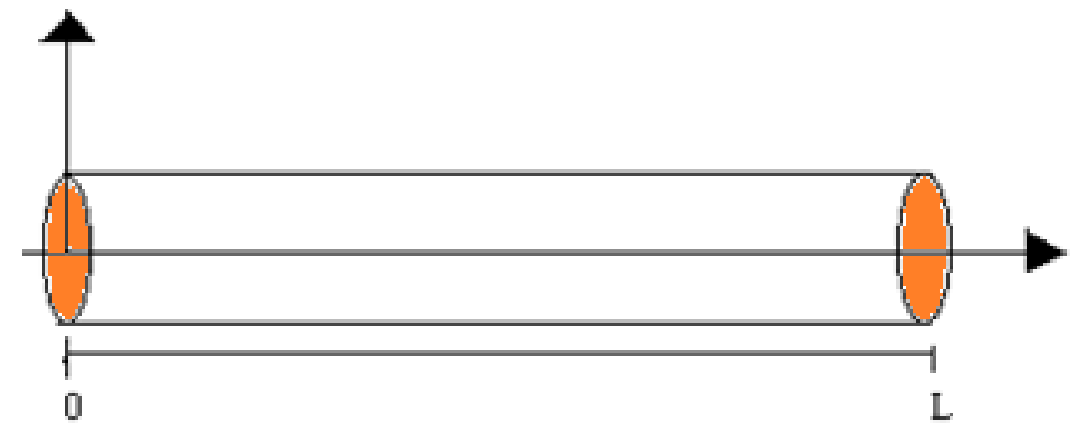

Fonte: elaboração dos autores.

A equação diferencial que descreve a condução de calor, com variação de temperatura nas extremidades nula, é descrita pela Equação 04.

$$
\begin{aligned}
& \frac{\partial u}{\partial t}=\alpha^{2} \frac{\partial^{2} u}{\partial x^{2}}, \quad(0<x<L, t>0) \\
& u_{x}(0, t)=u_{x}(L, t)=0, \quad t>0 \\
& u(x, 0)=f(x), \quad(0<x<L)
\end{aligned}
$$

Em que $\alpha$ é o coeficiente de difusividade do material.

\subsection{Softwares Maple e Excel}

Para equacionamento e resolução da função condução de calor, para o problema estudado, foi utilizado o software Maple, o qual é de fácil manuseio e de grande capacidade para implementar e resolver problemas compostos por equações algébricas dos mais complexos níveis.

De acordo com Muniz e Marczak:

O software MAPLE consiste em um sistema de computação algébrica. Formalmente, computação algébrica (as vezes chamada de manipulação algébrica ou computação 
simbólica) pode ser definida como a computação com variáveis e constantes de acordo com as regras da álgebra, análise e outros ramos da matemática. Realiza manipulação de expressões que envolvem símbolos, variáveis e operações formais, de preferência a trabalhar com dados convencionais, na forma de números e strings de caracteres. Resumidamente, o MAPLE é um software matemático cuja característica principal é a possibilidade de trabalhar com informação na forma algébrica. (MUNIZ; MARCZAK, 2001, p. 471).

Já, para representação e análise gráfica, foi utilizado o software Excel, o qual é muito utilizado no mundo acadêmico, por ser de fácil manuseio e de grande capacidade.

De acordo com Battisti:

O Excel é um software para criação e manutenção de Planilhas Eletrônicas, que permite, além da manipulação de cálculos em planilhas, a inclusão de gráficos criados com base nos dados da planilha. Podem ser criadas planilhas de cálculos para orçamentos, previsões e planejamentos para investimentos futuros, diversos tipos de tabelas, controle de gastos entre outras funções. (BATTISTI, 2010, p. 07)

Como observado pelo autor, o Excel é um software de fácil operação com planilhas eletrônicas, sendo possível analisar resultados e representar gráficos diretamente em suas planilhas.

\section{METODOLOGIA}

Para a realização da pesquisa, foram feitas simulações em um laboratório de física do IFSul, Campus Passo Fundo, RS, pelos autores, sendo estes professores do instituto, em que foi aquecida e após resfriada, em temperatura ambiente, uma barra de cobre de espessura fina, de comprimento de 27,2 $\mathrm{cm}$, isolada nas extremidades por tijolos refratários e aquecida por uma lamparina. 0 experimento está exposto na Figura 2.

Figura 2: Realização do experimento em laboratório

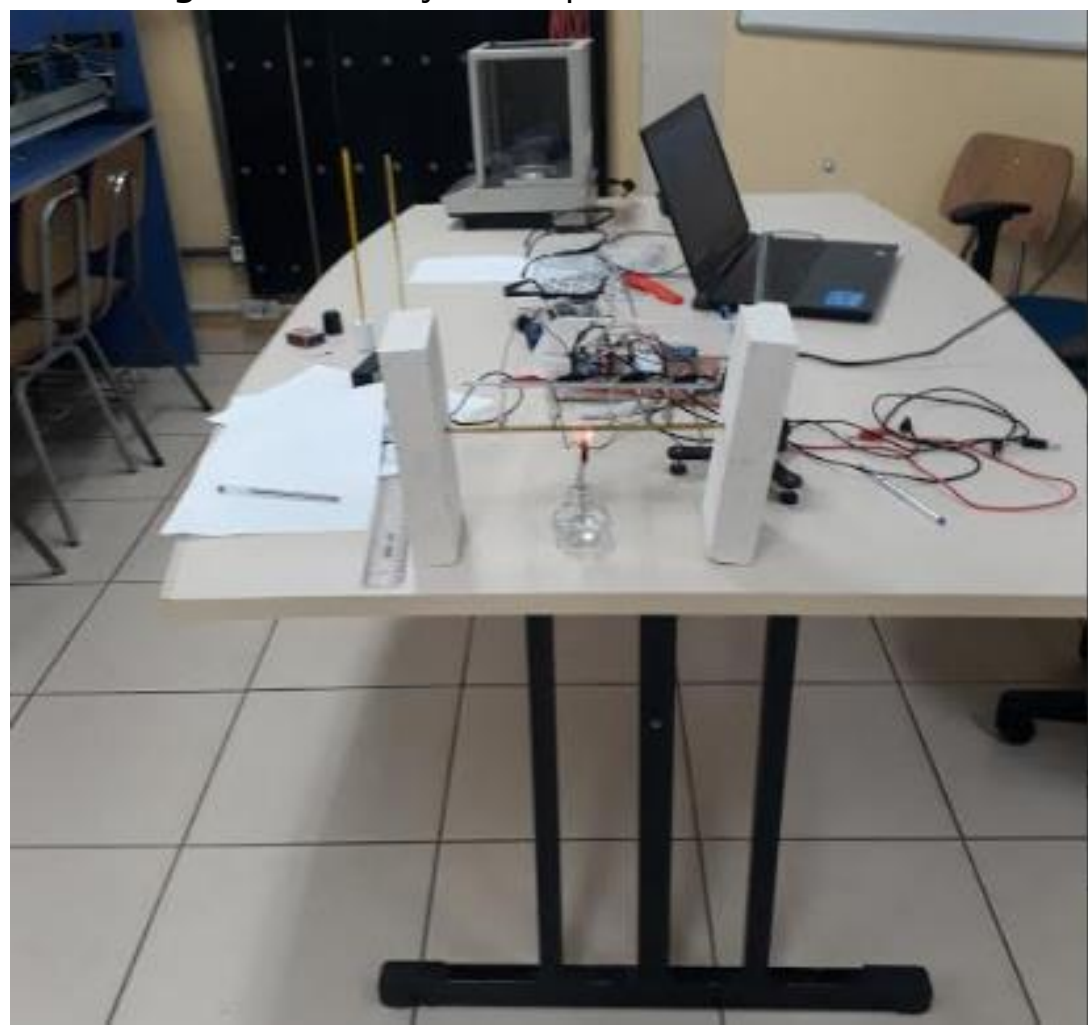

Fonte: elaboração dos autores. 
Para coleta de dados, foi retirada a lamparina e foram utilizados quatro sensores que coletavam a temperatura ao mesmo tempo, em intervalo de 17,5 segundos, à medida que a barra estava resfriando.

Considerando que há uma simetria de difusão de calor, com a fonte de calor centralizada, foi feita a análise de resfriamento somente de um lado da barra, sendo que, para o problema, foram coletados e plotados quatro valores, em função dos comprimentos $13,6 \mathrm{~cm}, 17 \mathrm{~cm}, 20,4 \mathrm{~cm}$ e 23,8 cm e dos tempos 0, 17,5 e 35 segundos. Conforme Figura 3 e Gráfico 1.

Figura 3: Comprimentos em que foram coletadas as temperaturas na barra de cobre

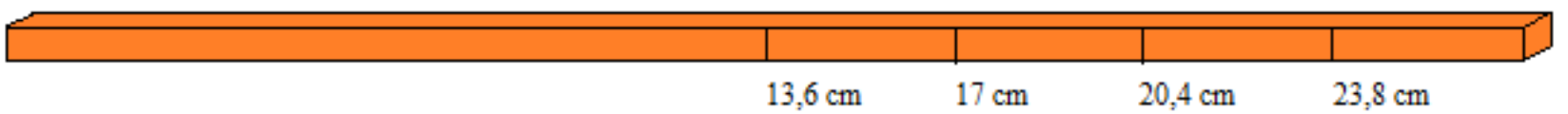

Fonte: elaboração dos autores.

Gráfico 1: Temperaturas coletadas pelos sensores em função dos comprimentos e dos tempos

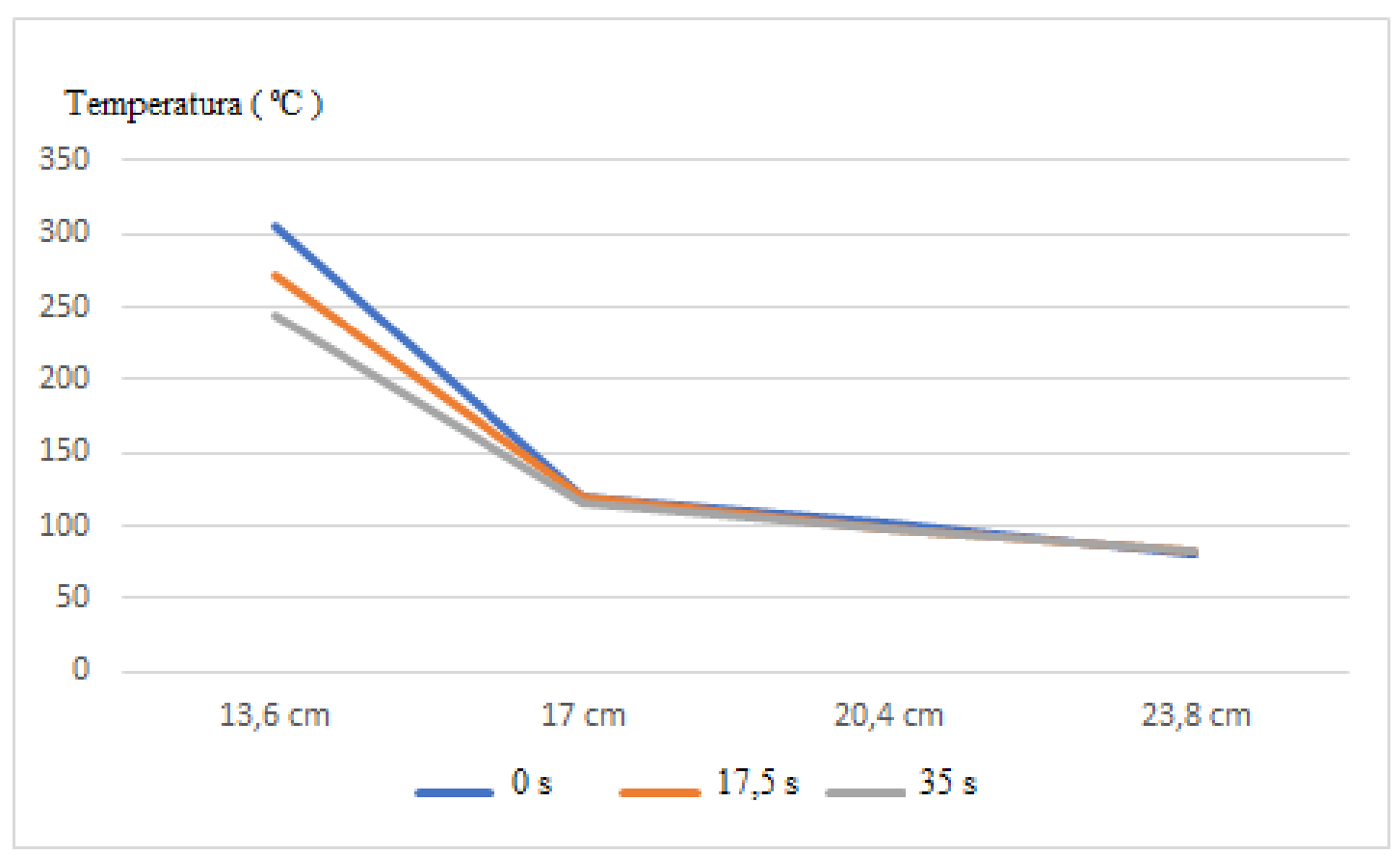

Fonte: elaboração dos autores.

Os valores obtidos, além de plotados, foram tabelados, conforme exposto na seção Resultados e discussões, para posterior comparação dos resultados alcançados via equação condução de calor.

Também, com esses resultados, foi possível obter a condição inicial, $u(x, 0)=f(x)$, para a equação diferencial, ou seja, o equacionamento da função temperatura no início do resfriamento, considerado o tempo igual a zero.

O problema se deu de acordo com a Equação 05.

$$
\begin{aligned}
& \frac{\partial u}{\partial t}=\alpha^{2} \frac{\partial^{2} u}{\partial x^{2}}, \quad(0<x<27,2 ; t>0) \\
& u_{x}(0, t)=u_{x}(27,2 ; t)=0, \quad t>0 \\
& u(x, 0)=f(x), \quad(0<x<27,2)
\end{aligned}
$$


Sendo que, no problema em questão, foi utilizada uma barra de cobre, cujo valor do coeficiente de difusividade, de acordo com a literatura, é 0,99, ou seja, $\alpha=0,99$.

Com a utilização de processos matemáticos, inclusive numéricos, foi obtida a equação condução de calor. Esta foi implementada no software Maple, obtendo, assim, a função condução de calor para o problema trabalhado (Equação 06), composta por uma série de onze termos.

$$
\begin{aligned}
u(x, t)=104.8 & -24.08397853 \cos (0.03676470588 \pi x) \mathrm{e}^{-0.001324745891 \pi^{2} t} \\
& -60.99160414 \cos (0.07352941176 \pi x) \mathrm{e}^{-0.001324745891 \pi^{2} t} \\
& +23.74645463 \cos (0.1102941176 \pi x) \mathrm{e}^{-0.001324745891 \pi^{2} t} \\
& +5.125982802 \cos (0.1470588235 \pi x) \mathrm{e}^{-0.001324745891 \pi^{2} t} \\
& -6.772070902 \cos (0.1838235294 \pi x) \mathrm{e}^{-0.001324745891 \pi^{2} t} \\
& -33.23661704 \cos (0.2205882353 \pi x) \mathrm{e}^{-0.001324745891 \pi^{2} t} \\
& +14.55765905 \cos (0.2573529412 \pi x) \mathrm{e}^{-0.001324745891 \pi^{2} t} \\
& +7.147300507 \cos (0.2941176470 \pi x) \mathrm{e}^{-0.001324745891 \pi^{2} t} \\
& -9.116259658 \cos (0.3308823529 \pi x) \mathrm{e}^{-0.001324745891 \pi^{2} t} \\
& -8.238112494 \cos (0.3676470588 \pi x) \mathrm{e}^{-0.001324745891 \pi^{2} t}
\end{aligned}
$$

Com $x$ sendo os comprimentos da barra e tos tempos utilizados no experimento.

Os valores expostos neste artigo, encontrados pela Equação (06), são correspondentes aos comprimentos 13,6 cm, $17 \mathrm{~cm}, 20,4 \mathrm{~cm}$ e 23,8 cm e aos tempos 0, 17,5 e 35 segundos, sendo estes plotados no Gráfico 2.

Gráfico 2: Temperaturas obtidas pela equação de condução de calor em função dos comprimentos e dos tempos

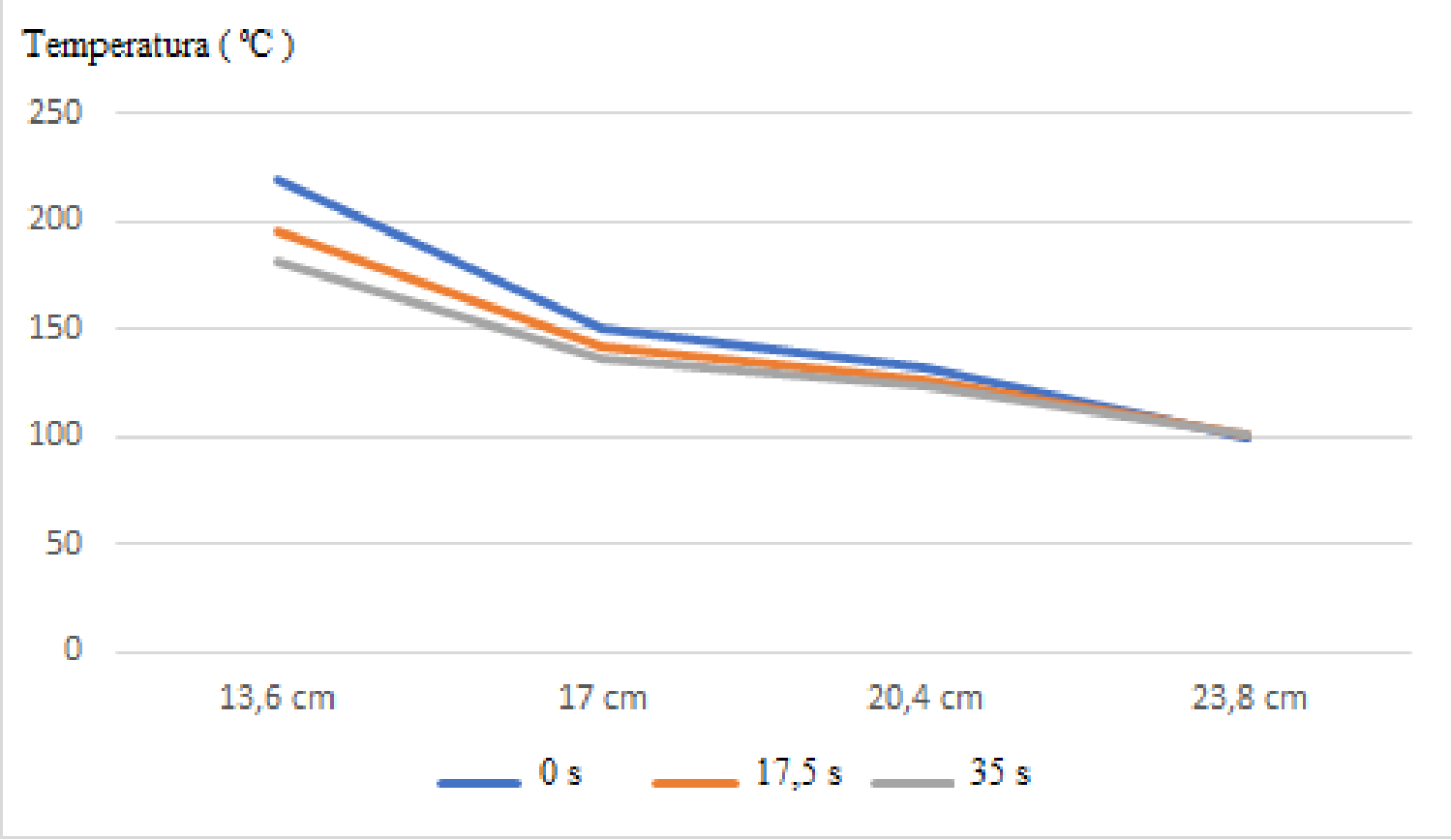

Fonte: elaboração dos autores. 


\section{RESULTADOS E DISCUSSÕES}

Após todo o processo que compõe a pesquisa, foi possível obter os valores da temperatura para o resfriamento da barra no laboratório, assim como os valores da temperatura para a barra através da equação condução de calor, em que foram comparados os resultados. Esses resultados estão expostos nas Tabelas 1 e 2, a seguir, e nos Gráficos 1 e 2, descritos na metodologia.

Tabela 1: Amostra dos dados obtidos via laboratório

\begin{tabular}{c|c|c|c}
\hline Compr./Tempo & $0 \mathrm{~s}$ & $17,5 \mathrm{~s}$ & $35 \mathrm{~s}$ \\
\hline $13,6 \mathrm{~cm}$ & 306 & 272 & 243,5 \\
\hline $17 \mathrm{~cm}$ & 120,5 & 118,75 & 115,75 \\
\hline $20,4 \mathrm{~cm}$ & 102,75 & 98,75 & 97 \\
\hline $23,8 \mathrm{~cm}$ & 80 & 81,75 & 82,25 \\
\hline
\end{tabular}

Fonte: elaboração dos autores.

Tabela 2: Amostra dos dados obtidos via equação do calor

\begin{tabular}{c|c|c|c}
\hline Compr./Tempo & $0 \mathrm{~s}$ & $17,5 \mathrm{~s}$ & $35 \mathrm{~s}$ \\
\hline $13,6 \mathrm{~cm}$ & 219,8 & 195,6 & 181,6 \\
\hline $17 \mathrm{~cm}$ & 150,9 & 141,33 & 135,7 \\
\hline $20,4 \mathrm{~cm}$ & 132 & 126,3 & 123 \\
\hline $23,8 \mathrm{~cm}$ & 99,4 & 100,5 & 101,2 \\
\hline
\end{tabular}

Fonte: elaboração dos autores.

Os resultados da Tabela 2 foram obtidos utilizando o par ordenado (comprimento, tempo) na Equação 06. Por exemplo, para a célula da linha três e coluna quatro, da Tabela 2 , foi feita uma $u(17,35)$, cujo resultado é 135,7 .

Os resultados se assemelham nos valores e no comportamento, somente para o tempo zero há uma diferença mais considerável.

Tais diferenças podem ser responsabilizadas pelas condições do experimento, como fonte de calor, isolamento das extremidades e temperatura ambiente, assim como a formação da equação condução de calor, sendo que ela é constituída por processo numérico com apenas onze termos.

\section{CONSIDERAÇÕES FINAIS}

A pesquisa apresentou resultados significantes ao realizar uma comparação entre os valores obtidos pelo experimento realizado no laboratório e os obtidos pela equação condução de calor, observandose uma proximidade nas temperaturas e no comportamento gráfico que descreve o problema.

Pesquisas comparativas entre resultados práticos e teóricos são de fundamental importância nas academias, pois levam aos pesquisadores um enriquecimento de conhecimentos, além de proporcionar recursos diferenciados para serem trabalhados em sala de aula, levando aos alunos uma abordagem diferenciada de estudos.

Considerando que é possível melhorar os resultados, tratamos esta pesquisa como inacabada e aberta para melhorias, com necessidade de aprimoramentos, tanto no experimento como na modelagem da equação. 
Já, ao considerar a viabilidade ou não de realização de uma tarefa prática envolvendo o assunto de equações diferenciais parciais, conclui-se que certamente é possível, pois proporcionará bons resultados para o problema.

Por ser viável a realização desta prática, a inserção de atividades laboratoriais nas aulas de cálculo certamente proporcionará aos alunos um novo método de absorção de conhecimento, além de tornar as aulas mais prazerosas e estimulantes para os envolvidos, ou seja, professores e alunos.

\section{REFERÊNCIAS}

BASSANEZI, Rodney Carlos. Modelagem matemática: teoria e prática. São Paulo: Contexto, 2015. BASSANEZI, Joni Rodney Carlos. Ensino-aprendizagem com modelagem matemática. 2. ed. São Paulo: Contexto, 2004.

BATTISTI, Júlio Cesar Fabris. Excel básico em 120 Lições. 2010. Disponível em: http://www.juliobattisti.com.br.pdf. Acesso em: 20 set. 2019.

DIACU, F. Introdução a equações diferenciais: teoria e aplicações. Rio de Janeiro: LTC, 2004.

FREIRE, Paulo. Pedagogia da autonomia: saberes necessários à prática educativa. São Paulo: Paz e Terra, 1996.

GOLDBARG, M. C.; LUNA, H. P. L. Otimização combinatória e programação linear: modelos e algoritmos. 2. ed. Rio de Janeiro: Elsevier, 2005.

HODSON, D. Hacia um enfoque más crítico del trabajo de laboratório. Enseñanza de las Ciencias, v. 12 , n. 13, p. 299-313, 1994.

INCROPERA, F. P. Z. M et al. Fundamentos de transferência de calor e de massa. Tradução e revisão técnica Eduardo Queiroz, Fernando Luiz Pellegrni Pessoa. Rio de Janeiro: LTC, 2008.

IÓRIO, V. M. EDP: um curso de graduação. Rio de Janeiro: Instituto de Matemática Pura e Aplicada, 2016. (Coleção Matemática Universitária).

LACORTT, M. Modelagem matemática para otimização do tráfego urbano semaforizado. 2011. Dissertação (Mestrado em Engenharia: Concentração Infraestrutura e Meio Ambiente) Faculdade de Engenharia e Arquitetura, Universidade de Passo Fundo, Passo Fundo, 2011.

LUNETTA, V. N. Atividades práticas no ensino da ciência. Revista Portuguesa de Educação, v. 2, n. 1, p. 81-90, 1992.

MELO, Kelly Jacqueline Moura de. Aplicação do método das diferenças finitas explícito na solução da equação do calor para o caso transiente e unidimensional. Monografia (Bacharel em Ciência e Tecnologia) - Universidade Federal Rural do Semi-Árido, Recife, 2011.

MUNIZ, André R.; MARCZAK, Lígia D. F. Uso do software Maple no ensino de transferência de calor. Cobenge, 2001.

NAGLE, R. K.; SAFF, E. B.; SNIDER, A. D. Equações diferenciais. 8. ed. São Paulo: Pearson, 2012.

Submissão: 30/04/2021

Aceito: 16/07/2021 